The invention disclosed in this document resulted from research in aeronautical and space activities performed under programs of the National Aeronautics and Space Administration. The invention is owned by NASA and is, therefore, available for licensing in accordance with the NASA Patent Licensing Regulation (14 Code of Federal Regulations 1245.2).

To encourage commercial utilization of NASA-owned inventions, it is NASA policy to grant licenses to commercial concerns. Although NASA encourages nonexclusive licensing to promote competition and achieve the widest possible utilization, NASA will consider the granting of a limited exclusive license, pursuant to the NASA Patent Licensing Regulations, when such a license, will provide the necessary incentive to the licensee to achieve early practical application to the invention.

Address inquiries and all applications fc license for this invention to NASA Patent Counsel, Ames Research Center, Mail Code 200-IlA, Moffett Field, California, 94035. Approved NASA forms for application for nonexclusive or exclusive license are available from the above address.

(NASA-C a Se-ARC-11925-1)

MULT I-VELOCITY COMPONENT LDV Patent Application (NASA. Ames Research Center) $19 \mathrm{p}$

$$
\text { D. I APRL }
$$

N95-14668

Unclas

ARC 
Title of the Invention

KULTI-VELOCITY COMPONENT LDV

\section{origin of the Invention}

The invention described herein was made by an employee of the U.S. Government and may be manufactured and used by or for the Government for governmental purposes without the payment of any royalties thereon or therefor.

\section{Background of the Invention}

Field of the Invention

The present invention relates generally to the field of laser velocimetry, and more specifically, to a laser doppler velocimeter (IDV) which uses frequency 20 shifting and a single channel digital frequency processor to effect multi-velocity component measurements.

25 Description of the Related Art

LDVs are now in common use for flow measurements during, for example, wind tunnel testing of various 30 structures. There are many types of known LDVs, but most operate off similar basic principles. 
Dual beam LDVs use optics to split a laser beam into two beams and to focus the beams to cross at a point where measurements are to be made. Due to wave interference, a fringe pattern is formed at the beam intersection. A second lens assembly, functioning as a receiver, is focused on the fringe region to collect light scattered from seed particles crossing the fringes. The collected light is fed to a photodetector that is used as the input to an electronic signal

10 processor that measures modulated frequency.

In a dual beam system the frequency from the scattered light from the split beams is directed onto the surface of the photodetector where it is mixed, and a difference in frequency between the two beams will

15 result.

Flow velocity can be derived from the modulated frequency, which can be expressed as $2 \mu_{x} \frac{\sin \beta}{\gamma}$ where $\mu_{x}=$ velocity parallel to the plane of the two beams and perpendicular to a bisector of the beams;

$\beta=$ angle between one of the beams and the bisector, and

$\gamma=$ wavelength of the laser 1 ight. An example of a signal processor for a LDV is described in U.S. Patent No. 4,786,168, issued to Meyers et al. on November 22, 1988. A processor is described therein for measuring the signal frequency within a signal burst. A photodetector converts an 30 optical signal, composed of scattered light emanating from the fringe pattern, into an electronic signal. 
The electronic signal is composed of a collection of Poisson distributed photo-electrons whose average occurrence rate is proportional to the instantaneous light intensity at the photocathode. As the intensity

5 increases from the photon resolved regime, the additional photon arrivals within the response time add voltage to the output signal. Eventually, the signal approaches a Gaussian-shaped signal burst containing the oscillation frequency. The frequency is multiplied by the distance between adjacent fringes to yield the velocity of the particle.

The aforementioned processor of Meyers et al. converts the input signal to digital and shifts it into shift registers. A signal integration circuit determines when a signal burst has been captured by the shift registers and transfers the contents to data latches, whereupon the data is processed by bandpass filters, square law detectors, burst counters and a signal processor to determine the frequency of the 20 signal.

W. Farmer and J. Hornkohl describe a LDV which measures two-vector components of velocity in an article entitled "Two-Component, self-Aligning Laser Vector Velocimeter", Applied optics, vol. 12, no. 11 (Nov. 1973). The LDV, schematically illustrated in Fig. 1, includes a laser 10 outputting a beam to a twodimensional Bragg cell 12. The Bragg cell 12 is actually two Bragg cells in a common housing arranged so that the center lines of the cells are coincident and orthogonal. This arrangement allows the Bragg cell 
12 to be both a beam splitter and a frequency shifter. The crystal oscillators of the Bragg cell 12 are driven at two different frequencies for detection separation of the respective velocity components. The Bragg cell 12 produces four output beams, three of which are diffracted beams and the fourth is the original input beam. The diffracted beams can be independently shifted up or down in frequency. The beams are focused by a lens 14 to cross simultaneously at a point. Scattered light is collected by a lens system 16 and focused on a photomultiplier 18 and the output signal therefrom is processed by signal processors 20 (only one shown) which use band-pass filters 21 to separate the carrier frequencies for the respective velocity components.

other methods of obtaining simultaneous multivelocity component LDV measurements involve, instead of frequency shifting, the use of a different laser wavelength for each velocity component. Generally, 20 multiple photodetectors and signal processors are used with narrow-band light filters placed ahead of the photodetectors. The use of multiple processors, either counter-type or digital frequency-type in LDV measurements, with one provided for each velocity component, is fairly common.

It is generally accepted in the laser doppler velocimeter field that a separate signal processor channel is needed for each velocity component signal under sparce seeding conditions. High speed digital counters facilitate this procedure by measuring the 
time between zero crossings of signals produced from individual particles passing through the LDV sensing volume. However, the requirement for multiple processing channels and photo-detectors makes the overall set up of an LDV using different wavelength (color) lasers complicated and expensive.

\section{Bummary of the Invention}

An object of the present invention is to provide a LDV which requires only a single channel signal processor for either two and three-velocity component 15 measurements.

Another object of the present invention is to provide a LDV which requires fewer optical components, as compared to LDVs using multiple laser wavelengths, and thus provides a cost saving without sacrificing 20 quality of measurements. Another object of the present invention is to provide a LDV capable of producing accurate measurements even with low seed particle concentrations.

These and other objects are met by providing a laser doppler velocimeter for measuring multi-velocity component flow of a fluid having seed particles dispersed therein, including a laser outputting a beam, a modulator receiving the laser beam and outputting at

30 least two frequency modulated diffracted beams and a primary beam, each of the at least two diffracted beams corresponding to a velocity component and having a 
different frequency, a transmitting lens receiving and converging the at least two frequency modulated diffracted beams and the primary beam to cross at a cross-over region, a collection lens positioned to collect particle-scattered light from the cross-over region, said collection lens focusing collected particle-scattered light into a multi-mode fiber, a light detector connected to the multi-mode fiber and producing a composite electrical signal containing

10 information about each velocity component, an analog to digital converter for digitizing the composite electrical signal, and a processor for producing a discrete Fourier transform based on the digitized electrical signal, and including a peak frequency 15 corresponding to each velocity component.

These and other features and advantages of the laser doppler velocimeter and method will become more apparent with reference to the following detailed description and drawings.

\section{Brief Description of the Dravinge}

Fig. 1 is a perspective view of a known twovelocity component LDV using a Bragg cell;

Fig. 2 is a perspective view of a LDV according to the present invention;

Fig. 3 is a perspective view of a LDV according to a second embodiment of the present invention;

Fig. 4 is a time chart reproduction of a typical electrical signal produced by the light detector of 
either embodiment of Figs. 2 and 3;

Fig. 5 is a schematic, perspective view illustrating a laser beam arrangement capable of producing the electrical signal illustrated in Fig. 4; Fig. $6(a)$ is a discrete Fourier transform of the electrical signal of Fig. 4;

Figs. $6(\mathrm{~b})$ and $6(\mathrm{c})$ are divided portions of the Fourier transform of Fig. $6(a)$; and

Fig. 7 is a schematic view of the signal processor of the present invention.

\section{petalled Description of the Preferred Embodiments}

Referring to Fig. 2, a IDV according to the present invention is generally referred to by the numeral 22 and includes a laser 24 outputting a beam 26 which passes through a beam waist positioner lens 28

20 and continues to a two dimensional acousto-optic modulator 30, which could be a Bragg cell such as the one described with respect to Fig. 1.

The modulator 30 outputs a primary beam and at least two diffracted laser beams, all of which define 25 multiple orthogonal planes. The beams are then passed through a transmitting lens 32 which causes the beams to cross or intersect at a common point, thus defining a cross-over region.

As a result of the beam waist positioner lens 28 , 30 the waist of each of the laser beams is at the common point. This ensures minimum variation in fringe spacing in the crossover region. 
A collecting lens 34 or combination of lenses is positioned to collect particle-scattered light from the laser beam crossover region. The lens 34 focuses the scattered light into one end of a multi-mode optical fiber 36, which transmits the light to a light detector 38. The detector 38 outputs via line 40 an electrical signal that contains several different frequencies from which multi-velocity component information can be extracted with a digital frequency processor 42 (to be described in more detail below).

Referring to Fig. 3, another embodiment of a LDV according to the present invention is generally referred to by the numeral 23 and it includes all of the same elements of the embodiment of the LDV 22 of Fig. 2, except that a single-mode optical fiber 27 is disposed between the laser 24 and the modulator 30 . This embodiment is more portable than that of the embodiment of Fig. 2 , and requires the addition of a fiber-optic coupler 27a. The coupler 27 a permits much

20 of the laser beam energy to enter the optical fiber 27 at one end, while the beam waist positioner lens 28 is disposed at the other end. The lens 28 functions the same in both embodiments.

One of the features of the present invention is that there only needs to be one single-mode optical fiber 27. In most dual-color two-velocity component LDV systems, four single-mode fibers would be required, each having its own coupler. Couplers such as coupler $27 \mathrm{a}$ of Fig. 3 are expensive and adjustments thereof are time consuming. Moreover, with multiple fibers, 
measurement errors can result because of variations in optical path lengths of the individual fibers, which can vary independently with time as a result of either mechanical stressing or non-uniform temperature exposure. Thus, the use of a single fiber avoids the aforementioned potential sources of measurement error.

To explain the signal processing according to the present invention, a representative electrical signal produced by the light detector 38 (for either

10 embodiment) is illustrated in Fig. 4. The signal, produced when a sub-micron size particle passes through the crossing laser beams 1llustrated in both Figs. 2 and 3 , contains information on two velocity components and results from the laser beams being frequency

15 shifted by the modulator 30. The signal illustrated in Fig. 4 could be produced by a laser beam arrangement illustrated in Fig. 5. Three laser beams 26a, 26b and 26c are produced by driving the two-dimensional acousto-optic modulator 30 at two different frequencies 20 fo(1) and $f o(2)$ and then blocking all but the primary beam $26 \mathrm{c}$ and two Bragg diffracted beams $26 \mathrm{a}$ and $26 \mathrm{~b}$. The velocity components sensed, $u_{1}$ and $u_{2}$ are substantially orthogonally, and each lies in the plane of a primary/diffracted laser beam pair and

25 perpendicular to corresponding bisectors b1 and b2 defined by each laser pair $(26 a, 26 c)$ and $(26 c, 26 b)$, respectively. A third velocity component is sensed by the two diffracted laser beams $26 \mathrm{a}$ and $26 \mathrm{~b}$, and 1 ies in the plane defined by the velocity components $u 1$ and $u 2$ 30 and is thus not linearly independent of $u 1$ and $u 2$. 
The frequency offset for this diagonal pair is either fo(1) + fo(2) or fo(1) - fo(2). The part of the signal due to this laser pair, which provides no additional velocity information, can easily be removed by electronic filtering and was done so for the signal in Fig. 4.

Measurement of a third independent velocity component could be accomplished by unblocking a third diffracted beam emanating from the acousto-optic modulator so that four laser beams instead of three intersect at the common point.

A discrete Fourier transform of the electrical signal of Fig. 4 is illustrated in Fig. 6(a). The resulting two peaks in the spectrum correspond to the 15 frequencies:

$$
\begin{aligned}
& f p(1)=f o(1)+f d(u 1)-f m, \text { and } \\
& f p(2)=f o(2)-f d(u 2)-f m \text {. }
\end{aligned}
$$

In the example illustrated in Fig. 4, the frequency has been reduced by downmixing at a frequency fm. The frequencies $f d(u 1)$ and $f d(u 2)$ are the Dopplershift frequencies associated with the velocity components $u 1$ and $u 2$. The sign of each of these two frequencies depends on whether the diffracted beam is at a lower or higher frequency than the primary beam 25 and on the sign convention adopted for the coordinate system. For the multi-frequency signal to be unambiguous, the acousto-optic frequencies fo(1) and fo(2) and the Doppler-shift sensitivities to $u 1$ and $u 2$ must be selected such that for all possible values of $u 1$ and $u 2$, $f p(1)>f c(1)>f p(2)$, where $f c(1)$ is a cut- 
off frequency which is predetermined. Existing commercially available laser Doppler velocimeter digital frequency processors can be modified to accommodate the spectrum of Fig. 6(a). The discrete Fourier transform produced by the processor, say that of Fig. 6(a), for example, needs only to be broken into the two separate spectra illustrated in Figs. $6(\mathrm{~b})$ and $6(c)$, with the first spectra corresponding to the frequency range from $\mathrm{fmin}$ to $\mathrm{fc}(1)$ and the second

10 spectra other from $f(1)$ to fmax. (fmax should not be larger than $1 / 2$ of the sampling frequency in order to avoid alliasing.) For generality, the cut-off frequency fc(1) should be selectable by the user. once the composite spectrum is broken into two parts as shown in Figs. $6(b)$ and $6(c)$, standard procedures adapted in commercial laser Doppler velocimeter digital frequency processors can be applied. For each spectrum, the value of the peak frequency can be determined using the same weighting 20 procedures, and the same signal to noise ratio (SNR) validation criteria can be used. The two spectrums of Figs. $6(\mathrm{~b})$ and $6(\mathrm{c})$ can be thought of as coming from two different digital frequency processors running synchronously. The individual spectrums can either be 25 analyzed sequentially or in parallel. Various criteria regarding SNR and signal amplitude can be implemented as if two separate processors were being used. In the case of three velocity components, two cut-off frequencies, $f c(1)$ and $f c(2)$, would be selectable. The 30 original composite spectrum in this case would be 
broken into three spectra which could then be analyzed individually.

Referring to Fig. 7, the signal processor 42 of the present invention includes an $A / D$ converter 44 which converts the analog electrical signal from the light detector 38 to a digital signal. The digital signal is fed to a discrete Fourier transform generator 46. Thus, the time-domain expression of the signal represented in Fig. 4 is converted to a mathematical equivalent of the same signal in the frequency domain by the generator 46 to yield the Fourier transform illustrated in Fig. 6(a). Optionally, a display 47 can be provided for displaying the transform. A frequency analyzer/separator 48 , in the form of a computer with 15 appropriate software, receives the transform data from the generator 46 and identifies and quantifies the twovelocity component peak frequencies. The analyzer/ separator 48 , based on the input of $f(c) 1$, separates the spectrum into two parts, as illustrated in Figs. $6(b)$ and $6(c)$, which are analyzed individually to determine the peak frequencies for $u 1$ and $u 2$, respectively.

The signals produced by individual particles passing through the sensing volume, as produced by the 25 light detector, are not flltered to separate out the two constituent velocity signals with two processors as is done in the prior art. Instead, the composite signal containing information of the different velocity components is inputted to the same single-channel digital frequency processor. Because a different 
Ereguency ohift is applied to each velocity channel (as 1. conventionally done when frequency shlfting io uead for channel 1eolation), the different velooity component eignale are diatingulahable from their location in the frequency epectra.

Numerous modiflcations and adaptations of the present Invention w111 be apparent to those so sk111ed In the art and thue, it, in Intended by the following claims to cover all such modifloations and adaptations 10 which fall within the true epirit and scope of the Invontion. 


\section{Abstract}

A laser doppler velocimeter uses frequency shifting of a laser beam to provide signal information for each velocity component. A composite electrical signal generated by a light detector is digitized and a processor produces a discrete Fourier transform based on the digitized electrical signal. The transform includes two peak frequencies corresponding to the two velocity components. 

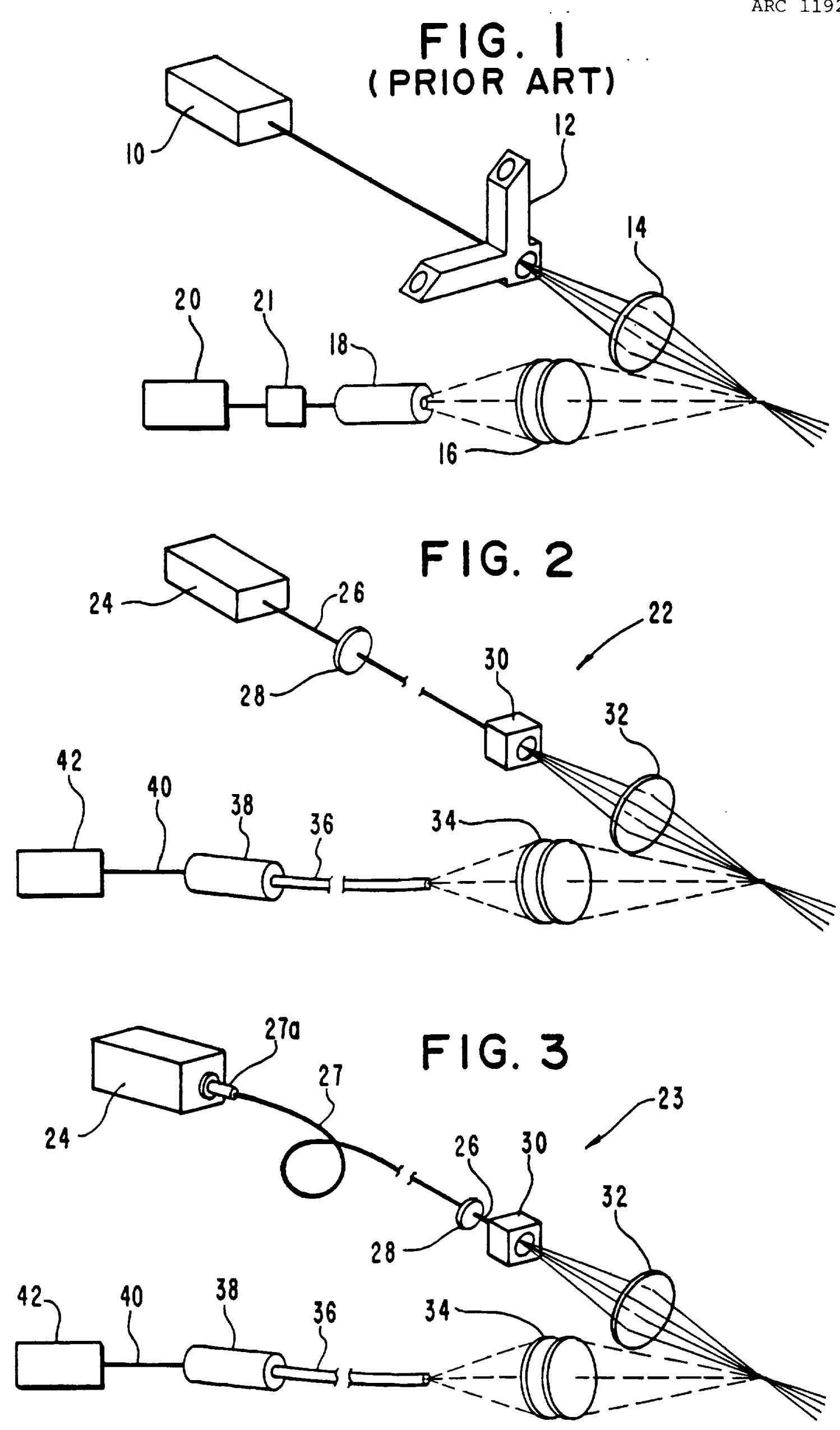
F |G. 4

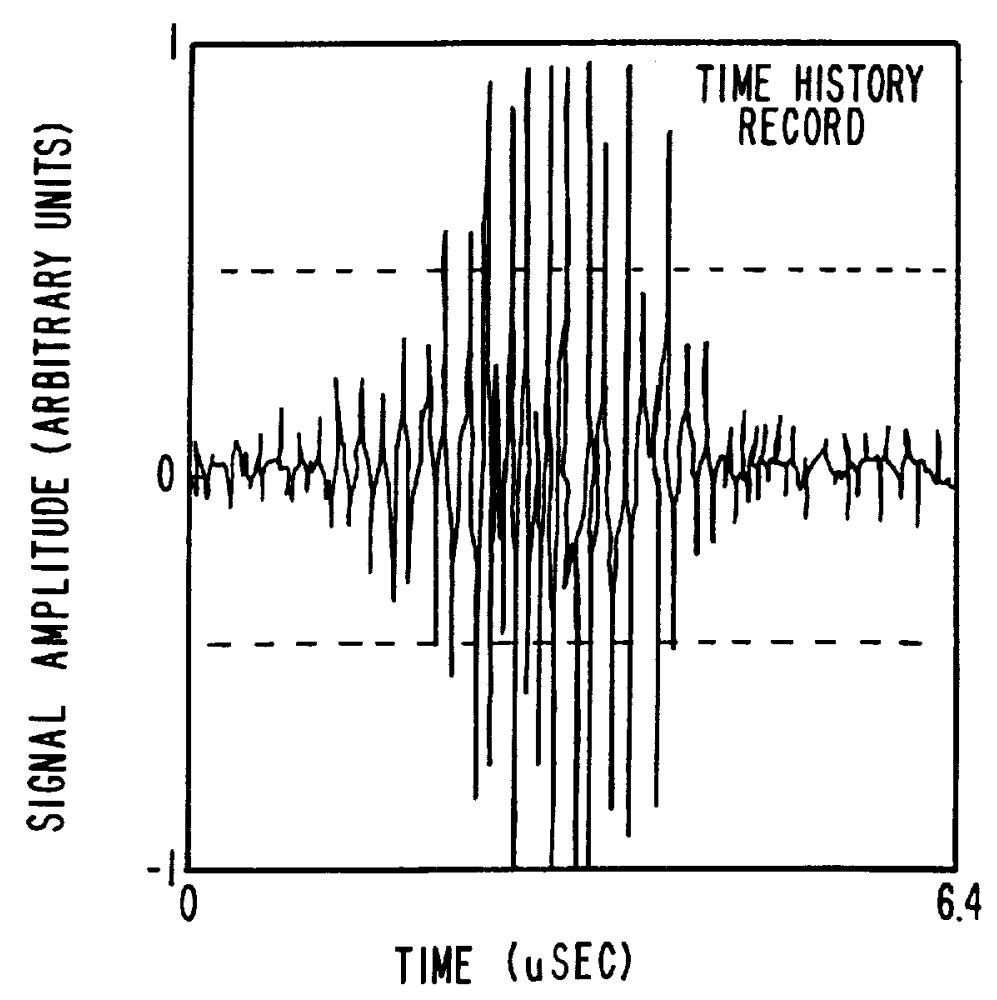

F | G. 5

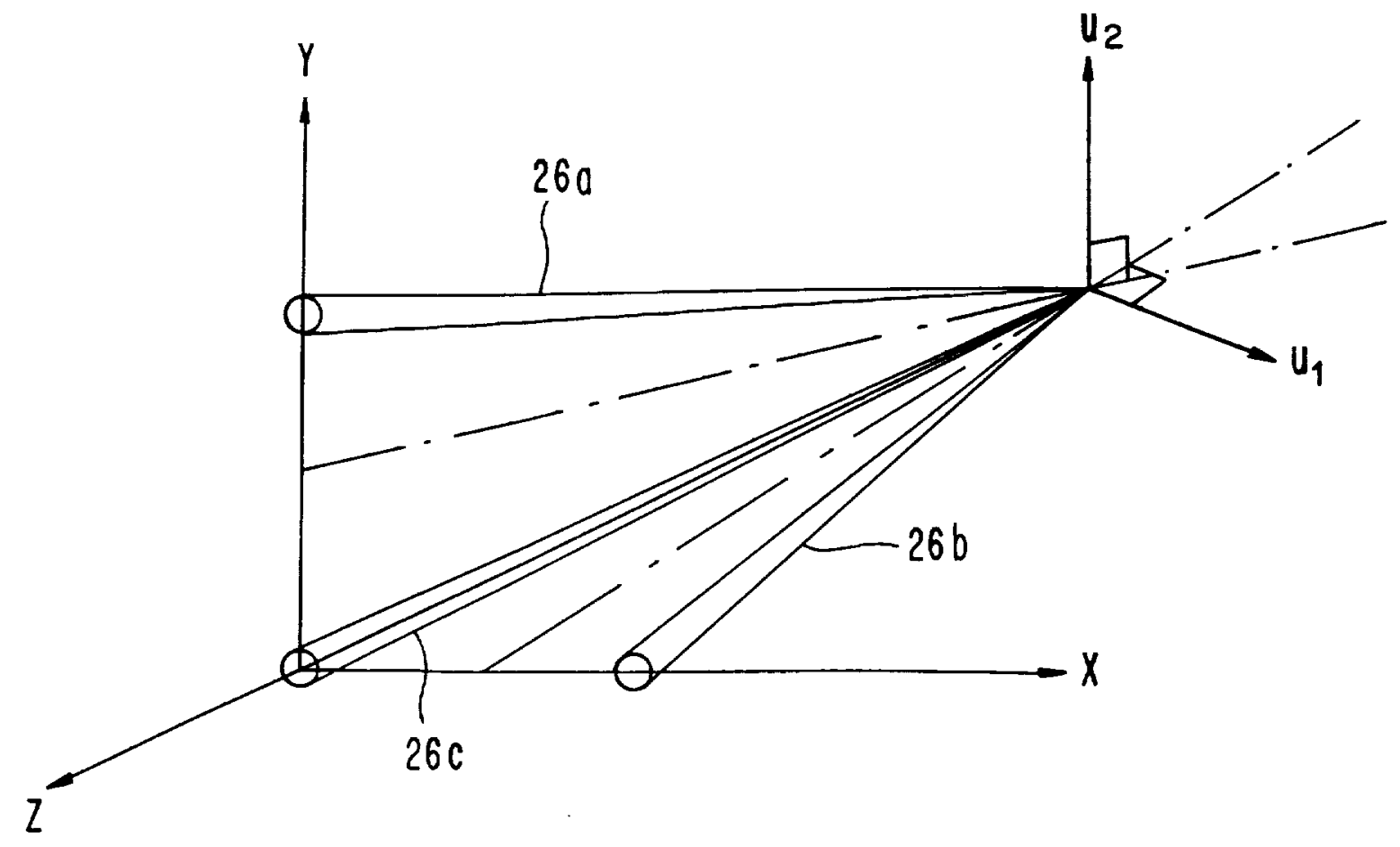



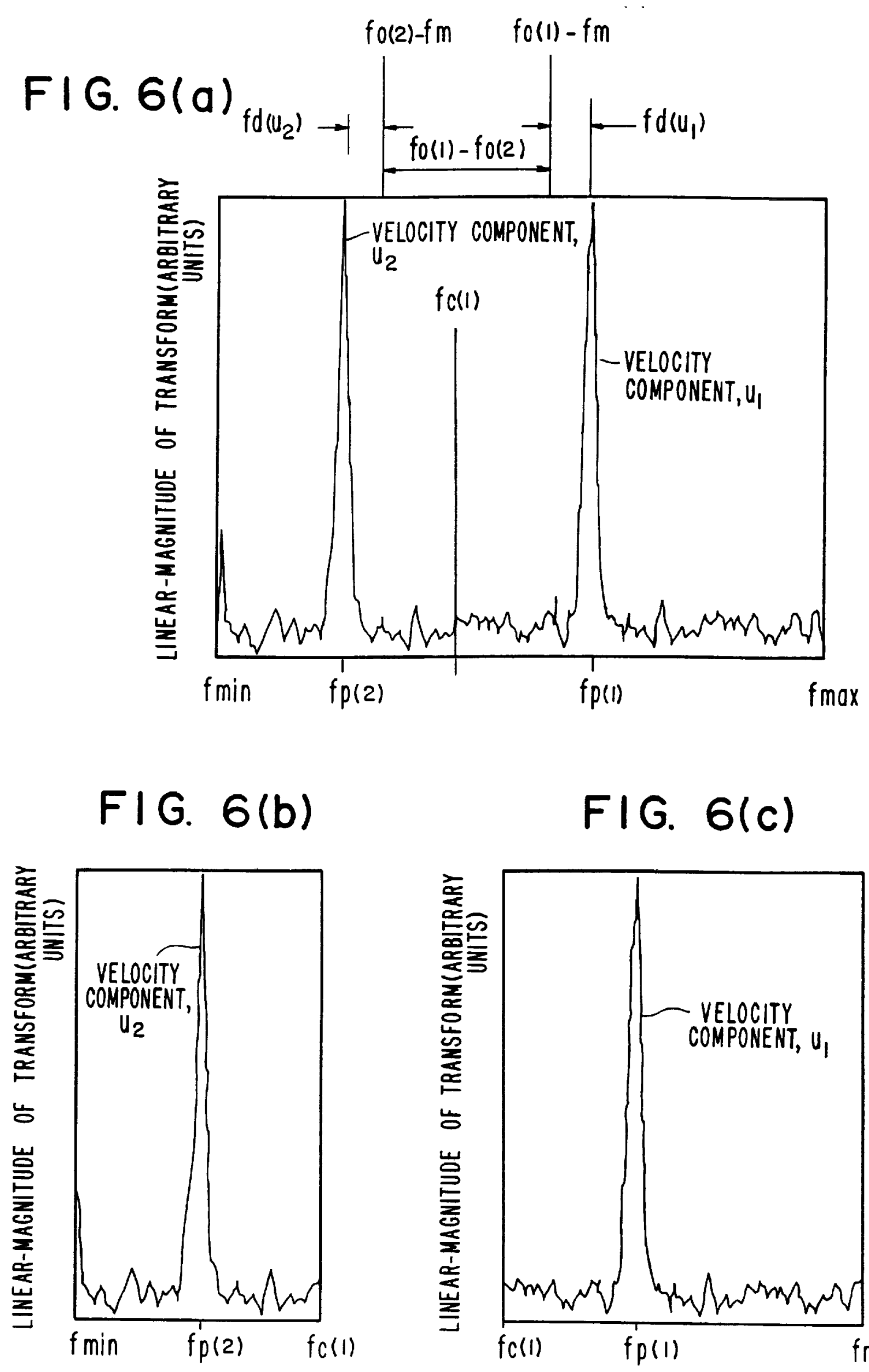

FIG. $6(c)$

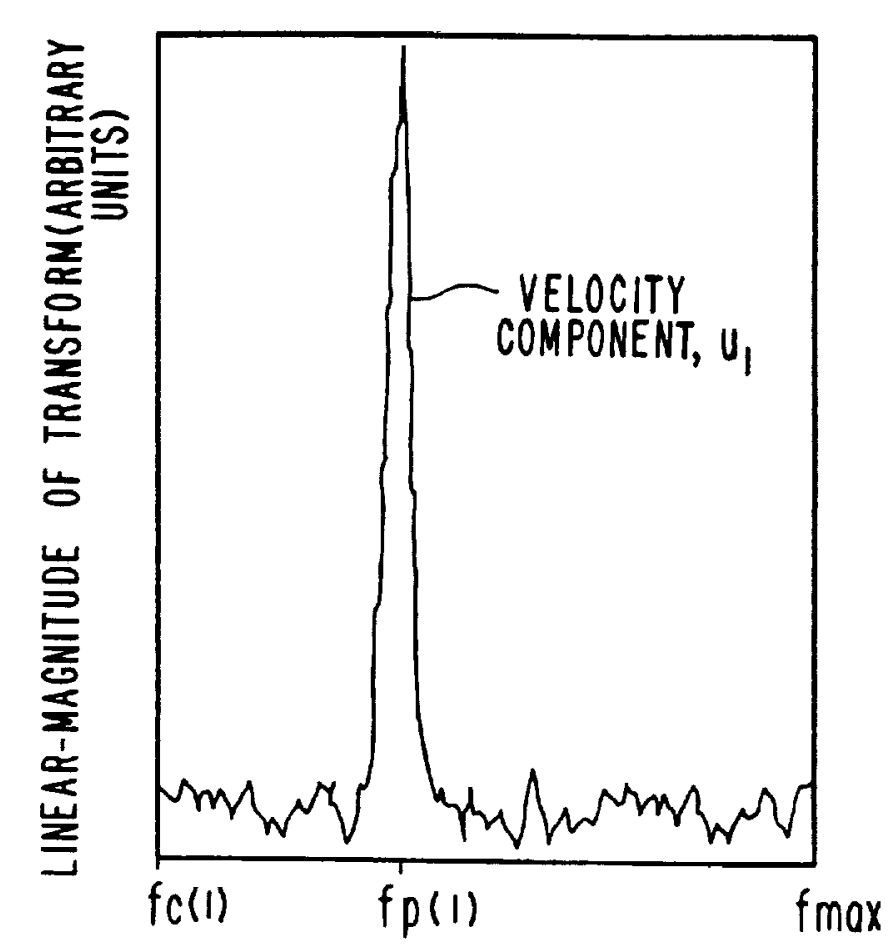


FIG. 7

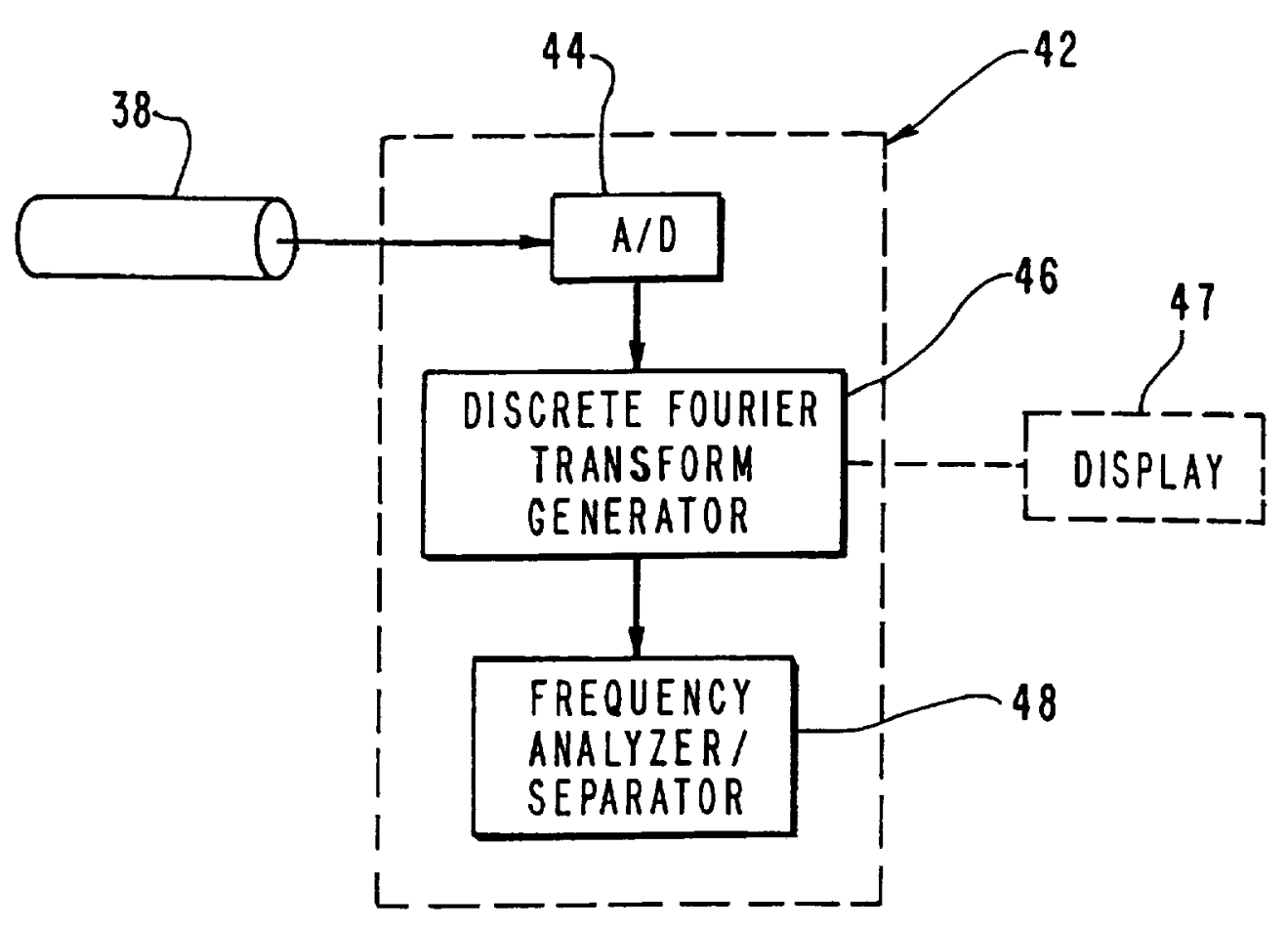

